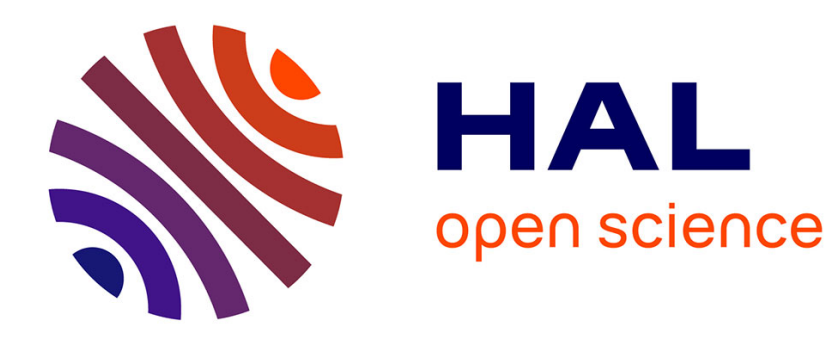

\title{
Robust stochastic control and equivalent martingale measures
}

Bernt Oksendal, Agnès Sulem

\section{To cite this version:}

Bernt Oksendal, Agnès Sulem. Robust stochastic control and equivalent martingale measures. [Research Report] RR-7557, INRIA. 2011, pp.11. inria-00573117

\section{HAL Id: inria-00573117 https://hal.inria.fr/inria-00573117}

Submitted on 3 Mar 2011

HAL is a multi-disciplinary open access archive for the deposit and dissemination of scientific research documents, whether they are published or not. The documents may come from teaching and research institutions in France or abroad, or from public or private research centers.
L'archive ouverte pluridisciplinaire HAL, est destinée au dépôt et à la diffusion de documents scientifiques de niveau recherche, publiés ou non, émanant des établissements d'enseignement et de recherche français ou étrangers, des laboratoires publics ou privés. 


\title{
Robust stochastic control and equivalent martingale measures
}

\author{
Bernt Øksendal — Agnès Sulem
}

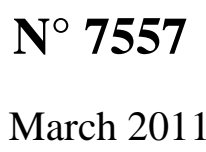

Stochastic Methods and Models

\section{apport}

de recherche 



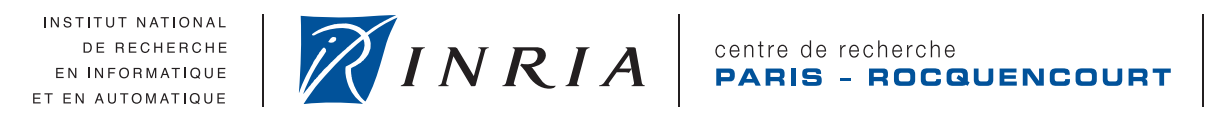

\title{
Robust stochastic control and equivalent martingale measures
}

\author{
Bernt Øksenda: $⿴$, Agnès Sulem \\ Theme : Stochastic Methods and Models \\ Équipe-Projet Mathfi \\ Rapport de recherche $\mathrm{n}^{\circ} 7557$ - March 2011 - 11] pages
}

\begin{abstract}
We study a class of robust, or worst case scenario, optimal control problems for jump diffusions. The scenario is represented by a probability measure equivalent to the initial probability law. We show that if there exists a control that annihilates the noise coefficients in the state equation and a scenario which is an equivalent martingale measure for a specific process which is related to the control-derivative of the state process, then this control and this probability measure are optimal. We apply the result to the problem of consumption and portfolio optimization under model uncertainty in a financial market, where the price process $S(t)$ of the risky asset is modeled as a geometric Itô-Lévy process. In this case the optimal scenario is an equivalent local martingale measure of $S(t)$. We solve this problem explicitly in the case of logarithmic utility functions.
\end{abstract}

Key-words: robust control, model uncertainty, worst case scenario, portfolio optimization, Lévy Market

\footnotetext{
* Center of Mathematics for Applications (CMA), Dept. of Mathematics, University of Oslo, P.O. Box 1053 Blindern, N-0316 Oslo, Norway, email: oksendal@math.uio.no and Norwegian School of Economics and Business Administration, Helleveien 30, N-5045 Bergen, Norway. The research leading to these results has received funding from the European Research Council under the European Community's Seventh Framework Programme (FP7/2007-2013) / ERC grant agreement no [228087]

$\dagger$ INRIA Paris-Rocquencourt, Domaine de Voluceau, Rocquencourt, BP 105, Le Chesnay Cedex, 78153, France, email: agnes.sulem@inria.fr
} 


\section{Contrôle stochastique robuste et mesures martingales équivalentes}

Résumé : On étudie une classe de contrôle optimal robuste - ou pire des cas - pour des processus de duffusions avec sauts. Le scénario est représenté par une mesure de probabilité équivalente à la probabilité initiale. On montre que s'il existe un contrôle qui annule les coefficients aléatoires dans l'équation d'état et un scénario qui est une mesure martingale équivalente pour un certain processus associé à la dérivée de l'état par rapport au contrôle, alors ce contrôle et cette mesure de probabilité sont optimaux. On applique ce résultat à un problème de consommation et portefeuille optimal avec incertitude de modèle dans un modèle de Marché de Lévy. Dans ce cas le scénario optimal est une mesure martingale locale équivalente du processus de prix. Le problème est résolu explicitement dans le cas de fonctions d'utilité logarithmiques.

Mots-clés : contrôle robuste, incertitude de modèles, scenario pire des cas, portfolio optimization, Processus de Lévy 


\section{Introduction}

During the last decade there has been an increasing awareness of the importance of taking model uncertainty into account when dealing with mathematical models. See e.g. [HS and the references therein. A general feature of model uncertainty is the recognition of the uncertainty about the underlying probability law, or scenario, for the model. This leads to the study of robust models, where one seeks an optimal strategy among a family of possible scenarios. A special case is the problem of optimal control in the worst possible scenario. This is the topic of this paper. We consider a class of scenarios which is basically the set of all probability measures $Q$ which are absolutely continuous with respect to a given reference measure $P$, and we study an optimal control problem for a jump diffusion under the worst possible scenario.

Mathematically this leads to a stochastic differential game between the controller and the "environment" who chooses the scenario. Assuming the system is Markovian and using the Hamilton-Jacobi-Bellman-Isaacs (HJBI) equation, we show that if there exists a control $\hat{u}$ which annihilates the noise coefficients of the system and a scenario $\hat{Q}$ which is an equivalent martingale measure for a specific process which is related to the controlderivative of the state process, then this control is optimal for the controller and the scenario $\hat{Q}$ is a worst case scenario.

We then apply this result to the problem of optimal consumption and portfolio under model uncertainty in a financial market, where the price process $S(t)$ of the risky asset is modeled as a geometric Itô-Lévy process. In this case the optimal scenario is an equivalent local martingale measure of $S(t)$. We solve this problem explicitly in the case of logarithmic utility functions.

Robust control problems and worst case scenario problems have been studied by many researchers. We mention in particular the paper [BMS], where the following approach is used: The authors first fix a strategy and prove the existence of a corresponding optimal scenario $Q^{*}$, and then subsequently use BSDEs to study the optimal strategy problem for a fixed scenario. The advantage with this approach is that it applies to nonMarkovian systems. Robust control problems for possibly non-Markovian systems can also be studied by means of stochastic maximum principles (see $\mathrm{A} \varnothing$ ).

\section{Robust optimal control}

\subsection{Stochastic differential game approach}

Consider a controlled jump diffusion $X(t)=X^{u}(t)$ in $\mathbb{R}$ of the form

$$
\begin{aligned}
d X(t) & =b(t, X(t), u(t)) d t+\sigma(t, X(t), u(t)) d B(t) \\
& +\int_{\mathbb{R}_{0}} \gamma(t, X(t), u(t), z) \tilde{N}(d t, d z) ; X(0)=x \in \mathbb{R} ; t \in[0, T]
\end{aligned}
$$

where $T>0$ is a fixed constant. Here $\tilde{N}(d t, d z)=N(d t, d z)-\nu(d z) d t$ is the compensated Poisson random measure of a Lévy process with jump measure $N(\cdot, \cdot)$ and Lévy measure $\nu(\cdot)$, and $B(t)$ is an independent Brownian motion. Both $\tilde{N}(\cdot, \cdot)$ and $B(\cdot)$ live on a filtered probability space $\left(\Omega, \mathcal{F},\left\{\mathcal{F}_{t}\right\}, P\right)$.

The process $u(t)=\left(u_{1}(t), \cdots, u_{m}(t)\right) \in \mathbb{R}^{m}$ is the control process of the agent and $b(s, x, u), \sigma(s, x, u)$ and $\gamma(s, x, u, z) ; s \in[0, T], x \in \mathbb{R}, u \in \mathbb{R}^{m}, z \in \mathbb{R}_{0}:=\mathbb{R} \backslash\{0\}$, are assumed to be $\mathcal{C}^{1}$ functions with respect to $u$.

The scenario of $X(t)$ is determined by a (positive) measure $Q^{\theta}$ of the form

$$
d Q^{\theta}(\omega)=K^{\theta}(T) d P(\omega) \text { on } \mathcal{F}_{T}
$$

$\mathrm{RR} \mathrm{n}^{\circ} 7557$ 
where

$$
\begin{aligned}
d K^{\theta}(t) & =-K^{\theta}\left(t^{-}\right)\left[\theta_{0}(t) d B(t)+\int_{\mathbb{R}_{0}} \theta_{1}(t, z) \tilde{N}(d t, d z)\right] ; t \in[0, T] \\
K^{\theta}(0) & =k>0 .
\end{aligned}
$$

Here $\theta=\theta(t, z)=\left(\theta_{0}(t), \theta_{1}(t, z)\right) \in \mathbb{R}^{2}$ is the scenario control, assumed to be $\mathcal{F}_{t}$-predictable and such that

$$
E\left[K^{\theta}(T)\right]=K^{\theta}(0)=: k>0 .
$$

Let $V, \Theta$ be two sets such that $u(t) \in V$ and $\theta(t, z) \in \Theta$ for all $t, z$ and let $\mathcal{U}, \mathcal{A}$ be given families of admissible $u$-controls and $\theta$-controls, respectively.

Define the process $Y(t)=Y^{\theta, u}(t):=\left(K^{\theta}(t), X^{u}(t)\right)$. Then $Y(t)$ is a controlled jump diffusion with generator

$$
\begin{aligned}
A^{\theta, u} \varphi(t, y) & =A^{\theta, u} \varphi(t, k, x) \\
& =b(t, x, u) \frac{\partial \varphi}{\partial x}+\frac{1}{2} k^{2} \theta_{0}^{2} \frac{\partial^{2} \varphi}{\partial k^{2}}+\frac{1}{2} \sigma^{2}(t, x, u) \frac{\partial^{2} \varphi}{\partial x^{2}} \\
& -\theta_{0} k \sigma(t, x, u) \frac{\partial^{2} \varphi}{\partial k \partial x} \\
& +\int_{\mathbb{R}_{0}}\left\{\varphi\left(t, k-k \theta_{1}(z), x+\gamma(t, x, u, z)\right)-\varphi(t, k, x)\right. \\
& \left.+k \theta_{1}(z) \frac{\partial \varphi}{\partial k}(t, k, x)-\gamma(t, x, u, z) \frac{\partial \varphi}{\partial x}(t, k, x)\right\} \nu(d z) ; \varphi \in \mathcal{C}^{1,2,2}\left(\mathbb{R}^{3}\right) .
\end{aligned}
$$

We refer to ØS1 for more information about stochastic control of jump diffusions.

Let $f: \mathbb{R}^{2} \times V \rightarrow \mathbb{R}$ and $g: \mathbb{R} \rightarrow \mathbb{R}$ be functions such that

$$
E_{Q^{\theta}}\left[\int_{0}^{T}|f(t, X(t), u(t))| d t+|g(X(T))|\right]<\infty
$$

for all $u \in \mathcal{U}, \theta \in \mathcal{A}$. Define the performance functional by

$$
\begin{aligned}
J^{\theta, u}(t, y) & =E_{Q^{\theta}}^{t, y}\left[\int_{t}^{T} f(s, X(s), u(s)) d s+g(X(T))\right] \\
& =E^{t, y}\left[\int_{t}^{T} K^{\theta}(s) f(s, X(s), u(s)) d s+K^{\theta}(T) g(X(T))\right],
\end{aligned}
$$

where $E_{Q^{\theta}}^{t, y}$ and $E^{t, y}$ denotes expectation with respect to $Q^{\theta}$ and $P$, respectively, given $Y(t)=y$.

We consider the following robust, or worst case scenario, stochastic control problem

Problem 2.1 Find $\theta^{*} \in \mathcal{A}, u^{*} \in \mathcal{U}$ and $\Phi(t, y)$ such that

$$
\Phi(t, y)=\inf _{\theta \in \mathcal{A}}\left(\sup _{u \in \mathcal{U}} J^{\theta, u}(t, y)\right)=J^{\theta^{*}, u^{*}}(t, y)
$$




\subsection{The main theorem}

We now formulate our main result:

Theorem 2.2 Suppose there exist a $\mathcal{C}^{1}$ function $\psi(t, x)$ and feedback controls $\hat{u}=\hat{u}(t, x) \in \mathcal{U}, \hat{\theta}=\left(\hat{\theta}_{0}(t, x), \hat{\theta}_{1}(t, x, z)\right) \in$ $\mathcal{A}$ such that

$$
\sigma(t, x, \hat{u}(t, x))=\gamma(t, x, \hat{u}(t, x), z)=0 \text { for all } t, x, z
$$

and

$$
\begin{aligned}
& {\left[\hat{\theta}_{0}(t, x) \frac{\partial \sigma}{\partial u_{i}}(t, x, \hat{u}(t, x))+\int_{\mathbb{R}_{0}} \hat{\theta}_{1}(t, x, z) \frac{\partial \gamma}{\partial u_{i}}(t, x, \hat{u}(t, x), z) \nu(d z)-\frac{\partial b}{\partial u_{i}}(t, x, \hat{u}(t, x))\right] \frac{\partial \psi}{\partial x}(t, x)} \\
& =\frac{\partial f}{\partial u_{i}}(t, x, \hat{u}(t, x)) ; \text { for all } t, x, \quad i=1,2, \ldots, m .
\end{aligned}
$$

Then $(\hat{u}, \hat{\theta})$ is an optimal pair for the robust control problem (2.7) and the value function is given by $\Phi(t, k, x)=$ $k \psi(t, x)$; provided that $\psi(t, x)$ is the solution of the PDE

$$
\begin{gathered}
\frac{\partial \psi}{\partial t}(t, x)+b(t, x, \hat{u}(t, x)) \frac{\partial \psi}{\partial x}(t, x)+f(t, x, \hat{u}(t, x))=0 ;(t, x) \in[0, T] \times \mathbb{R} \\
\psi(T, x)=g(x) ; x \in \mathbb{R} .
\end{gathered}
$$

Proof. We apply Theorem 3.2 in MØ]. Maximizing $u \rightarrow A^{\theta, u} \varphi(t, k, x)+k f(t, x, u)$ with respect to $u$ gives the following first order conditions for an optimal $\hat{u}$ :

$$
\begin{aligned}
& \frac{\partial b}{\partial u_{i}}(t, x, \hat{u}) \frac{\partial \varphi}{\partial x}+\sigma(t, x, \hat{u}) \frac{\partial \sigma}{\partial u_{i}}(t, x, \hat{u}) \frac{\partial^{2} \varphi}{\partial x^{2}}-\theta_{0} k \frac{\partial \sigma}{\partial u_{i}}(t, x, \hat{u}) \frac{\partial^{2} \varphi}{\partial k \partial x} \\
& \quad+\int_{\mathbb{R}_{0}}\left\{\frac{\partial \varphi}{\partial x}\left(t, k-k \theta_{1}(z), x+\gamma(t, x, \hat{u}, z)\right)-\frac{\partial \varphi}{\partial x}(t, k, x)\right\} \frac{\partial \gamma}{\partial u_{i}}(t, x, \hat{u}, z) \nu(d z) \\
& \quad+k \frac{\partial f}{\partial u_{i}}(t, x, \hat{u})=0 ; \quad i=1,2, \ldots, m .
\end{aligned}
$$

Minimizing $A^{\theta, \hat{u}} \varphi(t, k, x)+k f(t, x, \hat{u})$ with respect to $\theta=\left(\theta_{0}, \theta_{1}(z)\right)$, we get the following first order conditions for optimal $\hat{\theta}_{0}, \hat{\theta}_{1}(z)$ :

and

$$
k^{2} \hat{\theta}_{0} \frac{\partial^{2} \varphi}{\partial k^{2}}-k \sigma(t, x, \hat{u}) \frac{\partial^{2} \varphi}{\partial k \partial x}=0
$$

$$
\int_{\mathbb{R}_{0}}\left\{\frac{\partial \varphi}{\partial k}\left(t, k-k \hat{\theta}_{1}(z), x+\gamma(t, x, \hat{u}, z)\right)-\frac{\partial \varphi}{\partial k}(t, k, x)\right\} \nu(d z)=0 .
$$

Let us try a value function of the form

$$
\varphi(t, k, x)=k \psi(t, x)
$$


Then (2.12)-(2.14) get the form

$$
\begin{gathered}
\frac{\partial b}{\partial u_{i}}(t, x, \hat{u}) \frac{\partial \psi}{\partial x}+\sigma(t, x, \hat{u}) \frac{\partial \sigma}{\partial u_{i}}(t, x, \hat{u}) \frac{\partial^{2} \psi}{\partial x^{2}}-\hat{\theta}_{0} \frac{\partial \sigma}{\partial u_{i}}(t, x, \hat{u}) \frac{\partial \psi}{\partial x} \\
+\int_{\mathbb{R}_{0}}\left\{\left(1-\hat{\theta}_{1}(z)\right) \frac{\partial \psi}{\partial x}(t, x+\gamma(t, x, \hat{u}, z))-\frac{\partial \psi}{\partial x}(t, x)\right\} \frac{\partial \gamma}{\partial u_{i}}(t, x, \hat{u}, z) \nu(d z) \\
+\frac{\partial f}{\partial u_{i}}(t, x, \hat{u})=0 ; \quad i=1,2, \ldots, m, \\
\sigma(t, x, \hat{u}) \frac{\partial \psi}{\partial x}(t, x)=0,
\end{gathered}
$$

and

$$
\int_{\mathbb{R}_{0}}\{\psi(t, x+\gamma(t, x, \hat{u}, z))-\psi(t, x)\} \nu(d z)=0 .
$$

Suppose there exists a Markov control $\hat{u}=\hat{u}(t, x)$ such that

$$
\sigma(t, x, \hat{u}(t, x))=\gamma(t, x, \hat{u}(t, x), z)=0 \text { for all } z \in \mathbb{R}_{0} .
$$

Then (2.17)-(2.18) are satisfied, and (2.16) gets the form

$$
\begin{aligned}
& {\left[\hat{\theta}_{0}(t, x) \frac{\partial \sigma}{\partial u_{i}}(t, x, \hat{u})+\int_{\mathbb{R}_{0}} \hat{\theta}_{1}(t, x, z) \frac{\partial \gamma}{\partial u_{i}}(t, x, \hat{u}, z) \nu(d z)-\frac{\partial b}{\partial u_{i}}(t, x, \hat{u})\right] \frac{\partial \psi}{\partial x}(t, x)} \\
& \quad=\frac{\partial f}{\partial u_{i}}(t, x, \hat{u}) ; \quad i=1,2, \ldots, m .
\end{aligned}
$$

Suppose $\hat{u}, \hat{\theta}$ satisfy (2.19)-(2.20). Then by Theorem 3.2 in $[\mathrm{M \varnothing}$ we are required to have

$$
\frac{\partial \varphi}{\partial t}(t, y)+A^{\hat{\theta}, \hat{u}} \varphi(t, y)+k f(t, x, \hat{u})=0 ; t<T .
$$

By (2.5) and (2.19), this gives the equation

$$
\frac{\partial \psi}{\partial t}(t, x)+b(t, x, \hat{u}(t, x)) \frac{\partial \psi}{\partial x}(t, x)+f(t, x, \hat{u}(t, x))=0 ; t<T,
$$

with terminal condition

$$
\psi(T, x)=g(x) ; x \in \mathbb{R} .
$$

This completes the proof.

Remark 2.3 Note that $\hat{u}(t, x)$ and $\hat{\theta}(t, x)$ might depend on $\frac{\partial \psi}{\partial x}(t, x)$. Hence equation (2.10) is in general a nonlinear PDE in the unknown function $\psi$. 


\subsection{Equivalent local martingale measures}

The following definition is motivated by applications in mathematical finance:

Definition 2.4 Let $S(t)$ be an Itô-Lévy process of the form

$$
d S(t)=\alpha(t) d t+\beta(t) d B(t)+\int_{\mathbb{R}_{0}} \lambda(t, z) \tilde{N}(d t, d z)
$$

for predictable processes $\alpha(t), \beta(t), \lambda(t, z) ; t \in[0, T], z \in \mathbb{R}_{0}$.

A probability measure $Q$ on $\mathcal{F}_{T}$ is called an equivalent local martingale measure (ELMM) for $S(\cdot)$ if $Q \sim P$ (i.e. $Q \ll P$ and $P \ll Q$ ) and $\{S(t)\}_{t \in[0, T]}$ is a local martingale with respect to $Q$.

It is well-known (see e.g. ØS1, Theorem 1.31]) that a measure $Q^{\theta}$ of the form (2.2)-(2.4) with $k=1$ is an ELMM for $S(\cdot)$ if and only if

$$
\theta_{0}(t) \beta(t)+\int_{\mathbb{R}_{0}} \theta_{1}(t, z) \lambda(t, z) \nu(d z)=\alpha(t) ; t \in[0, T] .
$$

Suppose that $\frac{\partial \psi}{\partial x} \neq 0$. Then, in view of Definition 2.4 the measure $Q_{\hat{\theta}}$ of the form (2.2)-(2.4) with $k=1$, where $\hat{\theta}$ is a scenario control satisfying (2.9), is a ELMM for all the processes $G_{i}(t)$, given by

$$
\begin{aligned}
& d G_{i}(t):=\left[\frac{\partial b}{\partial u_{i}}(t, \hat{X}(t), \hat{u}(t))+\left(\frac{\partial \psi}{\partial x}(t, \hat{X}(t))\right)^{-1} \frac{\partial f}{\partial u_{i}}(t, \hat{X}(t), \hat{u}(t))\right] d t \\
& +\frac{\partial \sigma}{\partial u_{i}}(t, \hat{X}(t), \hat{u}(t)) d B(t)+\int_{\mathbb{R}_{0}} \frac{\partial \gamma}{\partial u_{i}}(t, \hat{X}(t), \hat{u}(t), z) \tilde{N}(d t, d z) ; \quad i=1,2, \ldots, m .
\end{aligned}
$$

where $\hat{u}(t)=\hat{u}(t, \hat{X}(t))$ and $\hat{X}(t)=X^{\hat{u}}(t) ; t \in[0, T]$.

\section{Example}

Suppose we have a financial market with a risk free asset with unit price $S_{0}(t)=1$ and a risky asset with unit price $S(t)$ given by

$$
\begin{aligned}
d S(t) & =S\left(t^{-}\right)\left[b_{0}(t) d t+\sigma_{0}(t) d B(t)+\int_{\mathbb{R}_{0}} \gamma_{0}(t, z) \tilde{N}(d t, d z)\right] ; t \in[0, T] \\
S(0) & >0
\end{aligned}
$$

where $b_{0}(t), \sigma_{0}(t)$ and $\gamma_{0}(t, z)$ are bounded deterministic functions, $\gamma_{0}(t, z)>-1$. If we apply a portfolio $\pi(t)$, representing the proportion of the total wealth $X(t)$ invested in the risky asset at time $t$ and a relative consumption rate $\lambda(t) \geq 0$, the corresponding wealth process $X(t)=X^{\lambda, \pi}(t)$ will have the dynamics

$$
\begin{aligned}
d X(t) & =\pi(t) X\left(t^{-}\right)\left[b_{0}(t) d t+\sigma_{0}(t) d B(t)+\int_{\mathbb{R}_{0}} \gamma_{0}(t, z) \tilde{N}(d t, d z)\right]-\lambda(t) X(t) d t ; t \in[0, T] \\
X(0) & =x>0
\end{aligned}
$$


We say that the pair $u=(\lambda, \pi)$ is an admissible control if $\lambda$ and $\pi$ are $\mathcal{F}$-predictable, $\lambda \geq 0, \pi(t) \gamma_{0}(t, z)>-1$ and $\int_{0}^{T}\left(\pi^{2}(t)+\lambda(t)+\int_{\mathbb{R}_{0}}\left|\log \left(1+\pi(t) \gamma_{0}(t, z)\right)\right| \nu(d z)\right) d t<\infty$ a .s. Note that under these conditions the unique solution $X(t)$ of (3.2) is given by

$$
\begin{aligned}
X(t)= & x \exp \left(\int_{0}^{t}\left\{\pi(s) b_{0}(s)-\lambda(s)-\frac{1}{2} \pi^{2}(s) \sigma_{0}^{2}(s)\right\} d s+\int_{0}^{t} \pi(s) \sigma_{0}(s) d B_{s}\right. \\
& +\int_{0}^{t} \int_{\mathbb{R}_{0}}\left(\log \left(1+\pi(s) \gamma_{0}(s, z)\right)-\pi(s) \gamma_{0}(s, z)\right) \nu(d z) d s \\
& +\int_{0}^{t} \int_{\mathbb{R}_{0}}\left(\log \left(1+\pi(s) \gamma_{0}(s, z)\right) \tilde{N}(d s, d z)\right) ; t \in[0, T]
\end{aligned}
$$

In particular, $X(t)>0$ for all $t \in[0, T]$.

Let $U_{1}:[0, T] \times[0, \infty) \rightarrow \mathbb{R}, U_{2}:[0, \infty) \rightarrow \mathbb{R}$ be two given $\mathcal{C}^{1}$ functions. We assume that $c \rightarrow U_{1}(t, c)$ and $x \rightarrow U_{2}(x)$ are strictly increasing, concave functions (utility functions) for all $t \in[0, T]$. We also assume that $c \mapsto \frac{\partial U_{1}}{\partial c}(t, c)$ is strictly decreasing and that $\lim _{c \rightarrow+\infty} \frac{\partial U_{1}}{\partial c}(t, c)=0$ for all $t \in[0, T]$. Put $x_{0}=\frac{\partial U_{1}}{\partial c}(t, 0)$ and define

$$
I(t, x)= \begin{cases}0 & \text { for } x \geq x_{0} \\ \left(\frac{\partial U_{1}}{\partial c}(t, \cdot)\right)^{-1}(x) & \text { for } 0 \leq x<x_{0}\end{cases}
$$

Define the absolute consumption rate at time $t$ by

$$
c(t)=\lambda(t) X(t) ; t \in[0, T] .
$$

Suppose the performance functional is given by

$$
\begin{aligned}
J^{\theta, \lambda, \pi}(t, y) & =E_{Q^{\theta}}^{t, y}\left[\int_{t}^{T} U_{1}(s, c(s)) d s+U_{2}(X(T))\right] \\
& =E^{t, y}\left[\int_{t}^{T} K^{\theta}(s) U_{1}(s, c(s)) d s+K^{\theta}(T) U_{2}(X(T))\right] .
\end{aligned}
$$

To solve the problem

$$
\Phi(t, y)=\inf _{\theta \in \mathcal{A}}\left(\sup _{(\lambda, \pi) \in \mathcal{U}} J^{\theta, \lambda, \pi}(t, y)\right)=J^{\theta^{*}, \lambda^{*}, \pi^{*}}(t, y)
$$

we apply Theorem 2.2 Thus we search for a solution $\hat{c}, \hat{\pi}, \hat{\theta}=\left(\hat{\theta}_{0}, \hat{\theta}_{1}\right)$ and $\psi(t, x)$ such that (2.8) and (2.9) hold, when $b(t, x, u)=\pi x b_{0}(t)-\lambda x, \sigma(t, x, u)=\pi x \sigma_{0}(t), \gamma(t, x, u, z)=\pi x \gamma_{0}(t, x, z)$ and $f(t, x, u)=U_{1}(t, c)$, $g(x)=U_{2}(x), u=(\lambda, \pi), c=\lambda x$.

We see that (2.8) holds with $\hat{\pi}=0$, for all $\hat{c}$. Writing $\left(\frac{\partial}{\partial u_{1}}, \frac{\partial}{\partial u_{2}}\right)=\left(\frac{\partial}{\partial \lambda}, \frac{\partial}{\partial \pi}\right)$, equation (2.9) becomes

$$
\frac{\partial \psi}{\partial x}(t, x)=\frac{\partial U_{1}}{\partial c}(t, \hat{c}(t, x))
$$

and

$$
\hat{\theta}_{0}(t, x) \sigma_{0}(t)+\int_{\mathbb{R}_{0}} \theta_{1}(t, x, z) \gamma_{0}(t, z) \nu(d z)=b_{0}(t) .
$$

$\mathrm{RR} \mathrm{n}^{\circ} 7557$ 
The equation (2.10)-(2.11) for $\psi$ gets the form

$$
\begin{aligned}
& \frac{\partial \psi}{\partial t}(t, x)-\hat{c}(t, x) \frac{\partial U_{1}}{\partial c}(t, \hat{c}(t, x))+U_{1}(t, \hat{c}(t, x))=0 ; t<T \\
& \psi(T, x)=U_{2}(x)
\end{aligned}
$$

which has the solution

$$
\psi(t, x)=U_{2}(x)+\int_{t}^{T}\left\{U_{1}(s, \hat{c}(s, x))-\hat{c}(s, x) \frac{\partial U_{1}}{\partial c}(s, \hat{c}(s, x))\right\} d s .
$$

In this case the processes $G_{i}$ defined in (2.24) are

$$
\begin{aligned}
d G_{1}(t) & =\hat{X}(t)\left[-1+\left(\frac{\partial \psi}{\partial x}(t, \hat{X}(t))\right)^{-1} \frac{\partial U_{1}}{\partial c}(t, \hat{c}(t, \hat{X}(t)))\right] d t=0 \\
d G_{2}(t) & =\hat{X}\left(t^{-}\right)\left[b_{0}(t) d t+\sigma_{0}(t) d B(t)+\int_{\mathbb{R}_{0}} \gamma_{0}(t, z) \tilde{N}(d t, d z)\right] \\
& =\frac{\hat{X}\left(t^{-}\right)}{S\left(t^{-}\right)} d S(t) .
\end{aligned}
$$

We conclude that the optimal scenario control is to choose $\hat{\theta}=\left(\hat{\theta}_{0}, \hat{\theta}_{1}\right)$ such that (3.8) holds, i.e. such that $Q_{\hat{\theta}}$ is an ELMM for $S(t)$.

The corresponding optimal portfolio is to choose $\hat{\pi}=0$ (no money in the risky asset). This is intuitively reasonable, because if the price process is a martingale, there is no money to be gained by investing in this asset. have

Finally, to find the optimal consumption rate $\hat{c}(t, x)$ we combine (3.7) and (3.10) : From and 3.73) we

$$
\hat{c}(t, x)=I\left(t, \frac{\partial \psi}{\partial x}(t, x)\right) .
$$

Differentiating (3.10) we therefore get

$$
\frac{\partial \psi}{\partial t}(t, x)=-U_{1}\left(t, I\left(t, \frac{\partial \psi}{\partial x}(t, x)\right)\right)+I\left(t, \frac{\partial \psi}{\partial x}(t, x)\right) \frac{\partial \psi}{\partial x}(t, x) ; t<T .
$$

This is a nonlinear first order partial differential equation in $\psi(t, x)$. Together with the terminal value (obtained from (3.10)

$$
\psi(T, x)=U_{2}(x)
$$

this determines $\psi(t, x)$ uniquely. To summarize, we have proved

Theorem 3.1 The optimal (i.e. worst case) scenario control for the problem (3.6) is to choose $\theta^{*}=\left(\hat{\theta}_{0}, \hat{\theta}_{1}\right)$ such that (3.8) holds, which is equivalent to saying that the measure $Q_{\hat{\theta}}$ is an ELMM for the price process $S(t)$ given by (3.1).

The optimal portfolio under this scenario is to choose $\hat{\pi}=0$ (no money in the risky asset). The optimal consumption rate $\hat{c}(t, x)$ under this scenario is given by (3.11), i.e.

$$
\frac{\partial U_{1}}{\partial x}(t, \hat{c}(t, x))=\frac{\partial \psi}{\partial x}(t, x)
$$

$\mathrm{RR} \mathrm{n}^{\circ} 7557$ 
where $\psi(t, x)$ is the solution of (3.12)-(3.13). The corresponding value function is, by (2.15),

$$
\Phi(t, k, x)=k \psi(t, x) .
$$

This is an extension of the result in ØS2.

A special case. To illustrate the content of Theorem 3.1 we consider the special case when $U_{1}$ and $U_{2}$ are logarithmic exponential utility functions, i.e.

$$
U_{1}(s, c)=U_{1}(c)=\ln c ; \quad U_{2}(x)=\lambda \ln x
$$

where $\lambda>0$ is constant. Then $U_{1}^{\prime}(c)=\frac{1}{c}$ and, by (3.3) $I(x)=\frac{1}{x}$. Therefore equation (3.12) gets the form

$$
\frac{\partial \psi}{\partial t}(t, x)=\ln \left(\frac{\partial \psi}{\partial x}(t, x)\right)+1 .
$$

Set

$$
h(t, x)=\psi(t, x)-t .
$$

Then

$$
\frac{\partial h}{\partial t}(t, x)=\ln \left(\frac{\partial h}{\partial x}(t, x)\right) .
$$

Set

$$
H(t, x)=\frac{\partial h}{\partial x}(t, x) .
$$

Then $H$ satisfies the nonlinear PDE:

$$
H(t, x) \frac{\partial H}{\partial t}(t, x)=\frac{\partial H}{\partial x}(t, x) ; t \leq T
$$

We assign the terminal condition

$$
H(T, x)=\frac{\lambda}{x}
$$

and try to solve this equation by setting

$$
H(t, x)=H_{1}(t) \frac{1}{x} .
$$

Substituting into (3.18) gives

$$
H_{1}^{\prime}(t)=-1 \text {. }
$$

Since $H_{1}(T)=\lambda$, this gives the solution

$$
H(t, x)=\frac{T-t+\lambda}{x}
$$

and hence

$$
h(t, x)=(T-t+\lambda) \ln x+C(t)
$$

for some function $C(t)$. This gives

$$
\psi(t, x)=(T-t+\lambda) \ln x+t+C(t)
$$

$\mathrm{RR} \mathrm{n}^{\circ} 7557$ 
and hence

$$
\frac{\partial \psi}{\partial t}(t, x)=-\ln x+C^{\prime}(t)+1
$$

while

$$
\ln \left(\frac{\partial \psi}{\partial x}(t, x)\right)=\ln (T-t+\lambda)-\ln x .
$$

Using (3.17), we get

$$
C^{\prime}(t)=\ln (T-t+\lambda)-1
$$

or

$$
C(t)=-(T-t+\lambda) \ln (T-t+\lambda)+(T-t+\lambda)-t+C_{0}
$$

Hence

$$
\psi(t, x)=(T-t+\lambda)[\ln x-\ln (T-t+\lambda)+1]+C_{0} .
$$

Requiring

leads to the condition

$$
\psi(T, x)=U_{2}(x)=\lambda \ln x
$$

$$
C_{0}=\lambda \ln \lambda-\lambda \text {. }
$$

Hence the solution is

$$
\psi(t, x)=(T-t+\lambda)[\ln x-\ln (T-t+\lambda)]+\lambda \ln \lambda+T-t
$$

and the optimal consumption rate is then

$$
\hat{c}(t, x)=\frac{x}{T-t+\lambda} .
$$

In particular, we see that the consumption increases with time, which makes sense, because a large early consumption reduces the growth for the whole remaining time period.

\section{References}

[AØ] T.T.K. An and B. øksendal: A maximum principle for stochastic differential games with partial information. J. Optim. Theory and Appl. 139 (2008), 463 - 483.

[BMS] G. Bordigoni, A. Matoussi and M. Schweizer: A stochastic control approach to a robust utility maximization problem. In F.E.Benth et al (editors): Stochastic Analysis and Applications. Proceedings of the Second ASbel Symposium, Oslo 2005. Springer 2007, pp. 135-151.

[HS] L.P. Hansen and T.J. Sargent: Robust control and model uncertainty. The American Economic Review, Vol. 91, no 2, (2001), pp. 60-66.

[MØ] S. Mataramvura and B. Øksendal: Risk minimizing portfolios and HJBI equations for stochastic differential games. Stochastics 80 (2008), 317-337.

[ØS1] B. Øksendal and A. Sulem: Applied Stochastic Control of Jump Diffusions. Second Edition, Springer 2007.

[ØS2] B. Øksendal and A. Sulem: A game theoretic approach to martingale measures in incomplete markets. Eprint, Dept. of Mathematics, University of Oslo 24/2006. Survey of Applied and Industrial Mathematics (TVP Publishers, Moscow), 15, (2008), 18-24. 


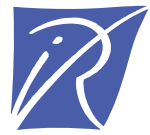

Centre de recherche INRIA Paris - Rocquencourt

Domaine de Voluceau - Rocquencourt - BP 105 - 78153 Le Chesnay Cedex (France)

Centre de recherche INRIA Bordeaux - Sud Ouest : Domaine Universitaire - 351, cours de la Libération - 33405 Talence Cedex

Centre de recherche INRIA Grenoble - Rhône-Alpes : 655, avenue de l'Europe - 38334 Montbonnot Saint-Ismier

Centre de recherche INRIA Lille - Nord Europe : Parc Scientifique de la Haute Borne - 40, avenue Halley - 59650 Villeneuve d'Ascq

Centre de recherche INRIA Nancy - Grand Est : LORIA, Technopôle de Nancy-Brabois - Campus scientifique

615, rue du Jardin Botanique - BP 101 - 54602 Villers-lès-Nancy Cedex

Centre de recherche INRIA Rennes - Bretagne Atlantique : IRISA, Campus universitaire de Beaulieu - 35042 Rennes Cedex

Centre de recherche INRIA Saclay - Île-de-France : Parc Orsay Université - ZAC des Vignes : 4, rue Jacques Monod - 91893 Orsay Cedex

Centre de recherche INRIA Sophia Antipolis - Méditerranée : 2004, route des Lucioles - BP 93 - 06902 Sophia Antipolis Cedex 